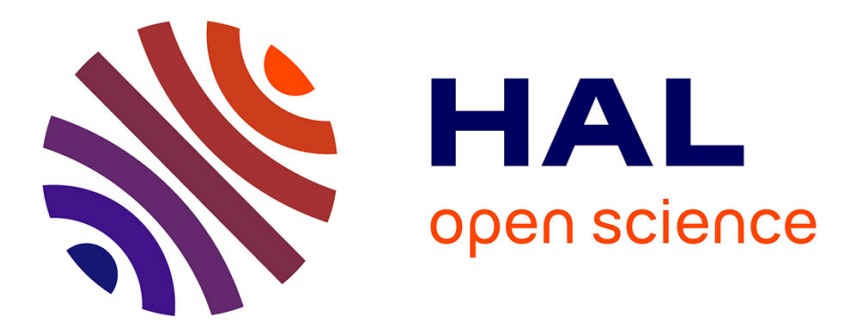

\title{
Strong Light Intensifications Yielded by Arbitrary Defects: Fresnel Diffraction Theory Applied to a Set of Opaque Disks
}

F. Tournemenne, S. Bouillet, C. Rouyer, C. Leymarie, J. Iriondo, B. da Costa Fernandes, G. Gaborit, B. Battelier, N. Bonod

\section{To cite this version:}

F. Tournemenne, S. Bouillet, C. Rouyer, C. Leymarie, J. Iriondo, et al.. Strong Light Intensifications Yielded by Arbitrary Defects: Fresnel Diffraction Theory Applied to a Set of Opaque Disks. Physical Review Applied, 2019, 11 (3), 10.1103/PhysRevApplied.11.034008 . hal-02398187

\section{HAL Id: hal-02398187 https://hal.science/hal-02398187}

Submitted on 6 Dec 2019

HAL is a multi-disciplinary open access archive for the deposit and dissemination of scientific research documents, whether they are published or not. The documents may come from teaching and research institutions in France or abroad, or from public or private research centers.
L'archive ouverte pluridisciplinaire HAL, est destinée au dépôt et à la diffusion de documents scientifiques de niveau recherche, publiés ou non, émanant des établissements d'enseignement et de recherche français ou étrangers, des laboratoires publics ou privés. 


\title{
Strong Light Intensifications Yielded by Arbitrary Defects: Fresnel Diffraction Theory Applied to a Set of Opaque Disks
}

\author{
F. Tournemenne, ${ }^{1,}{ }^{*}$ S. Bouillet, ${ }^{1}$ C. Rouyer, ${ }^{1}$ C. Leymarie,${ }^{1}$ J. Iriondo, ${ }^{1}$ B. Da Costa Fernandes, ${ }^{1}$ \\ G. Gaborit, ${ }^{1}$ B. Battelier, ${ }^{2}$ and N. Bonod ${ }^{3}$ \\ ${ }^{1}$ Commissariat à l'Energie Atomique et aux Energies Alternatives, Centre d'Etudes Scientifiques et Techniques \\ d'Aquitaine, 33116 Le Barp, France \\ ${ }^{2}$ LP2N, IOGS, CNRS and Université de Bordeaux, rue François Mitterrand, 33400 Talence, France \\ ${ }^{3}$ Aix-Marseille Univ, CNRS, Centrale Marseille, Institut Fresnel, Marseille, France
}

(Received 25 July 2018; revised manuscript received 5 December 2018; published 5 March 2019)

\begin{abstract}
Two centuries ago, Fresnel, Poisson, and Arago showed how the wave nature of light induces a bright spot behind an opaque disk. We develop an analytical model based on the Fresnel diffraction theory to show how small perturbations such as digs or scratches can yield intense light enhancements on the downstream axis. The impact of defects with complex morphology on light diffraction is shown to be accurately modeled by the Fresnel theory applied to a set of opaque disks characterized by phase shift and transmittance. This model can be used either to define the geometry of the defects that optimizes the light enhancement or to improve the defect specification on the surface of the optical components. We explain why partial information of the defect morphology can suffice to specify a safety distance beyond which light intensifications are not dangerous. The validity of this analytical approach is studied by measuring the intensifications created by three different microdefects.
\end{abstract}

DOI: 10.1103/PhysRevApplied.11.034008

\section{INTRODUCTION}

On 29 July 1818, A. Fresnel submitted to the French Academy of Sciences his seminal report on diffraction theory using the epigram Natura simplex et fecunda [1]. In order to refute this theory, S. D. Poisson predicted a counterintuitive effect: in the shadow of a light-blocking disk, a bright spot appears in its center. F. Arago experimentally proved the existence of this bright spot in 1819 (experiment reported in the first note added at the end of Fresnel's report) [1]. This bright spot, now called the Arago spot (sometimes the Poisson spot or the Fresnel bright spot) validated the wave nature of light. The light diffraction by an opaque disk yields two interesting results: (i) when the opaque mask has a circular shape, interference phenomena occur along the optical axis and (ii) when the mask impacts only light amplitude, interferences along the optical axis cannot yield strong light intensity enhancements.

Generally, defects in optical components feature much more complex shapes than the light-blocking disk considered in Arago's seminal experiment [2]. In this paper, we extend the concept of opaque disk diffraction by considering multiple disks that model defects with complex morphology. By applying the Babinet's principle $[3,4]$, the wave diffracted by defects is decomposed into a plane

\footnotetext{
*florian.tournemenne@cea.fr
}

wave and several phase-shifted plane waves propagating through a light-blocking disk.

A quantitative comparison with experimental defectinduced on-axis downstream light intensification is carried out in the framework of high-power beam lines. Diffraction patterns caused by the defects yield local high-field intensities that increase the probability of laser-induced damage [5]. Defects can occur from polishing processes [6], antireflective (AR) coating deposition, laser-induced damage mitigation [7], optics handling, contamination [8], or just from the interaction between light and the optical components [9].

Conventional methods used to determine defect-induced on-axis downstream light intensification consist of directly observing the impact of defects on diffracted light $[10,11]$. By using laser damage laws $[12,13]$, it is possible to predict the increase in the laser-induced damage probability for each observation of the diffracted intensities. The drawback of this empirical method is that the setup can be difficult to build and the process to evaluate the diffraction patterns created by each defect is time consuming.

Other methods are based on the simulation of light propagation through defects $[14,15]$. These numerical methods provide a lot of information about how defects impact light propagation. Moreover, some propagation codes include nonlinear propagation $[16,17]$, which, in certain conditions, must be considered to provide information on real 
defects' impacts on light propagation. The reliability of these numerical methods depends on the accuracy of the defect characterization. The phase shifts and the light transmittances through the defects have to be measured by interferential and photometric methods $[18,19]$. The characterization of defects is time consuming and difficult when such defects appear during the laser facility operation. When characterizations of the defects are missing, they are classically replaced by simple models, which can lead to false predictions.

Both experimental and numerical methods have spatial resolution limits and they can miss dangerous "hot spots." Indeed, light propagation can produce structures smaller than the resolution of the CCD camera or the mesh size of the simulation.

Here, we solve this issue by deriving an analytical method able to predict with a high accuracy defect-induced downstream light intensifications. Therefore, it can predict the generation of "hot spots" with small lateral sizes. We focus the study on the axis of the defects because we consider defects with circular symmetry for which the highest intensification tends to remain located along the optical axis. The key idea is to understand which defect parameters have to be characterized in order to find the safety area, which is defined by the distance after which intensifications cannot exceed a given threshold. The safety area, therefore, means that downstream optics mounted in this area cannot be damaged.

\section{FIELD DECOMPOSITION IN LIGHT-BLOCKING DISKS}

This section aims at modeling the intensifications generated by a defect model with an analytic approach based on wave decomposition into several elementary waves. This approach has been used in the microwave domain to model the effect of small phase objects in the near field $[20,21]$. For quasicircular objects, it has always been observed that the brightest spots occur along the optical axis of defects $[22,23]$. Therefore, this study is focused only on the light behavior along the optical axis. We will suppose in the whole study that the transverse dimensions are very large compared to the wavelength.

Defects are modeled by $m$ concentric rings. The $i$ th ring has a transmittance $t_{i}$, an outer radius $R_{i}$, and a phase $\phi_{i}$ (we use positive values for phase retardations). The substrate is assumed to have a transmittance $t_{m+1}$ and a uniform phase $\phi_{m+1}$, which is set to zero. The wave propagates along the $z$ axis. Then, the light wave perturbed by the rings structure, $U_{\text {str }}$, is represented as

$$
U_{\text {str }}(x, y, z=0)=\sum_{i=1}^{m+1} t_{i} e^{j \phi_{i}} \Upsilon_{i}(x, y, 0),
$$

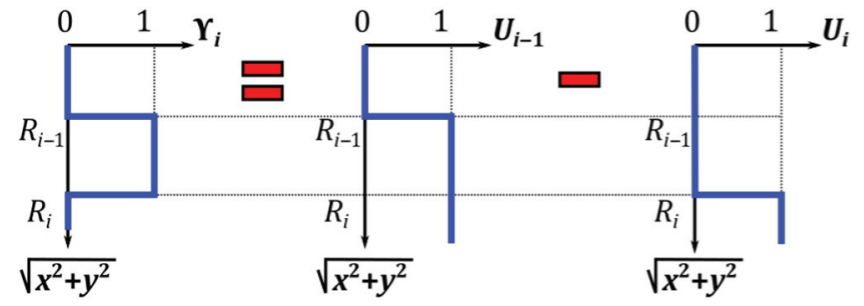

FIG. 1. Decomposition in $z=0$ of the $i$ th ring by two opaque disks: $\Upsilon_{i}(x, y, 0)=U_{i-1}(x, y, 0)-U_{i}(x, y, 0)$.

where $\left(\Upsilon_{i}\right)_{i \in[1, m+1]}$, are the elementary rings defined by

$$
\Upsilon_{i}(x, y, 0)= \begin{cases}1 & \text { if } \quad R_{i-1} \leq \sqrt{x^{2}+y^{2}}<R_{i} \\ 0 & \text { else }\end{cases}
$$

with $R_{0}=0$ and $R_{m+1}=\infty$.

Each ring is the difference between two opaque disks (see Fig. 1) defined by

$$
U_{i}(x, y, 0)= \begin{cases}0 & \text { if } \sqrt{x^{2}+y^{2}} \leq R_{i} \\ 1 & \text { else. }\end{cases}
$$

It can be observed that $\forall(x, y) \in \mathbb{R}^{2}, U_{0}(x, y, 0)=1$, and $U_{m+1}(x, y, 0)=0$.

Now, let us expand the structured field:

$$
\begin{aligned}
U_{\text {str }}(x, y, 0)= & \sum_{i=1}^{m+1}\left[U_{i-1}(x, y, 0)-U_{i}(x, y, 0)\right] t_{i} e^{j \phi_{i}} \\
= & \sum_{i=0}^{m} U_{i}(x, y, 0) t_{i+1} e^{j \phi_{i+1}} \\
& -\sum_{i=1}^{m+1} U_{i}(x, y, 0) t_{i} e^{j \phi_{i}} \\
= & t_{1} e^{j \phi_{1}}+\sum_{i=1}^{m}\left(t_{i+1} e^{j \phi_{i+1}}-t_{i} e^{j \phi_{i}}\right) U_{i}(x, y, 0) .
\end{aligned}
$$

As it is made with Babinet's principle [3,4], by using the linear property of light diffraction, the propagation of a field can be calculated from the sum of the diffraction of elementary fields. Let $\mathfrak{D}_{(x, y, z)}$ be the diffraction linear operator, which propagates the light wave to the point $(x, y, z)$. Applying $\mathfrak{D}_{(x, y, z)}$ to $U_{\text {str }}$ leads to

$$
\begin{aligned}
\mathfrak{D}_{(x, y, z)}\left[U_{\mathrm{str}}\right]= & t_{1} e^{j k z} e^{j \phi_{1}}+\sum_{i=1}^{m}\left(t_{i+1} e^{j \phi_{i+1}}-t_{i} e^{j \phi_{i}}\right) \\
& \times \mathfrak{D}_{(x, y, z)}\left[U_{i}\right]
\end{aligned}
$$


where $k=n k_{0}, n$ is the refractive index of the propagation medium, $k_{0}=2 \pi / \lambda$ is the wavenumber, and $\lambda$ is the wavelength.

The light wave diffracted by the structure is the sum of a $\phi_{1}$ phase-shifted plane wave and the diffraction of $m$ plane waves with complex amplitudes, which are diffracted by opaque disks.

The first Rayleigh-Sommerfeld diffraction in the Cartesian coordinates takes the form [24]

$$
\begin{aligned}
\mathfrak{D}_{(x, y, z)}\left[U_{i}\right]= & -\frac{j}{\lambda z} \iint_{\left(x^{\prime}, y^{\prime} \in \Sigma\right)} U_{i}\left(x^{\prime}, y^{\prime}, 0\right) \\
& \times \frac{e^{j k z \sqrt{1+\left(\frac{x-x^{\prime}}{z}\right)^{2}+\left(\frac{y-y^{\prime}}{z}\right)^{2}}}}{1+\left(\frac{x-x^{\prime}}{z}\right)^{2}+\left(\frac{y-y^{\prime}}{z}\right)^{2}} d x^{\prime} d y^{\prime},
\end{aligned}
$$

where $\Sigma$ is the plane where the $i$ th disk is located.

The Fresnel approximation is obtained by performing the Taylor expansion of the square root up to the first order in the exponential argument (numerator) and up to the zero order in the denominator. Along the optical axis, in the Fresnel approximation $\left(z \gg R_{i}\right)$, the diffracted wave is

$$
\begin{aligned}
\mathfrak{D}_{(0,0, z)}\left[U_{i}\right]= & -\frac{j}{\lambda z} e^{j k z} \iint_{\left(x^{\prime}, y^{\prime}\right) \in \Sigma} U_{i}\left(x^{\prime}, y^{\prime}, 0\right) \\
& \times e^{j \frac{k\left(x^{\prime 2}+y^{\prime 2}\right)}{2 z}} d x^{\prime} d y^{\prime} .
\end{aligned}
$$

By using the stationary phase theorem $[25,26]$, the diffraction integrals are calculated for a light-blocking disk with radius $R_{i}$ on the optical axis $\left(z \gg R_{i}\right)$ :

$$
\begin{aligned}
\mathfrak{D}_{(0,0, z)}\left[U_{\mathrm{ob}}\right] & =-\frac{j}{\lambda z} e^{j k z} \int_{R_{i}}^{+\infty} \int_{0}^{2 \pi} e^{j\left[k r^{2} /(2 z)\right]} r d r d \theta \\
& =e^{j\left[\pi R_{i}^{2} /(\lambda z)\right]} e^{j k z}
\end{aligned}
$$

Equation (8) shows that the intensification $\left|\mathfrak{D}_{(0,0, z)}\left[U_{\mathrm{ob}}\right]\right|^{2}$ is equal to 1 along the $z$ axis.

Now, the light wave diffracted by the structure [Eq. (5)] becomes

$$
\begin{aligned}
& \mathfrak{D}_{(0,0, z)}\left[U_{\mathrm{str}}\right] \\
& \quad=e^{j k z}\left[t_{1} e^{j \phi_{1}}+\sum_{i=1}^{m}\left(t_{i+1} e^{j \phi_{i+1}}-t_{i} e^{j \phi_{i}}\right) e^{j \pi \alpha_{i} N_{F}}\right],
\end{aligned}
$$

where $\left(\alpha_{i}\right)_{i \in[1, m]}$ is the sequence defined by

$$
\alpha_{i}=\left(R_{i} / R_{m}\right)^{2},
$$

and we use the notation conventionally used in the Fresnel diffraction theory $[27,28]$ :

$\left\{\begin{array}{l}Z_{c}=R_{m}^{2} / \lambda, \text { the characteristic propagation distance, } \\ N_{F}=Z_{c} / z, \text { Fresnel's number. }\end{array}\right.$

Let us point out that the Fresnel approximation $(z \gg R)$ is equivalent to the following statements: $z / Z_{c} \gg \lambda / R$ and $N_{F} \ll R / \lambda$.

The triangular inequality $\left[(a, b) \in \mathbb{C}^{2} \Rightarrow|a+b| \leq\right.$ $|a|+|b|]$ applied to Eq. (9) gives an upper bound to downstream intensifications:

$$
\begin{aligned}
I_{\text {str }} & \leq\left(t_{1}\left|e^{j \phi_{1}}\right|+\sum_{i=1}^{m}\left(t_{i+1}\left|e^{j \phi_{i+1}}\right|+t_{i}\left|e^{j \phi_{i}}\right|\right)\left|e^{j \alpha_{i} N_{F}}\right|\right)^{2} \\
& \leq\left(t_{m+1}+2 \sum_{i=1}^{m} t_{i}\right)^{2}
\end{aligned}
$$

Equation (12) shows that the highest intensification is generated by structures for which all the elements interfere constructively. It corresponds to the case of a pure phase object (transmittances equal to 1 ), which cannot generate higher intensifications than $(1+2 m)^{2}$. This limit is very large and, in most cases, it will not be reached. However, without more information than the number of disks $m$ used to model the defect, this limit is the lowest bound that can be defined. Lower limits can be obtained if information on phases, transmittances, and dimensions of the disks are obtained.

For example, for a pure phase object, the triangular inequality can be used to give a more subtle upper bound, which depends on the phase shifts:

$$
\begin{aligned}
I_{\text {str }} & =\left|e^{j \phi_{1}}+2 j \sum_{i=1}^{m} \sin \frac{\phi_{i+1}-\phi_{i}}{2} e^{j \frac{\phi_{i+1}+\phi_{i}}{2}} e^{j \pi \alpha_{i} N_{F}}\right|^{2} \\
& \leq\left(1+2 \sum_{i=1}^{m}\left|\sin \frac{\phi_{i+1}-\phi_{i}}{2}\right|\right)^{2} .
\end{aligned}
$$

Applying Eq. (13) to a structure with phase differences of $\pi$ leads to the intensification limit $I_{\text {str }} \leq(1+2 m)^{2}$. This result can be interpreted with the Fresnel zones [29]. Each zone is defined by all the point sources for which the propagation distance belongs to $[z+i \lambda / 2 ; z+(i+1) \lambda / 2]$, where $i$ is Fresnel's zone index (starting from zero). The contributions to the field of two consecutive Fresnel zones will cancel out. Therefore, the diffracted intensification will be increased by objects that introduce a phase delay or phase advance of $\pi$ for every other Fresnel zone. For instance, we represent in Fig. 2 the on-axis intensification diffracted by a $\pi$-phase disk and a $\pi$-phase ring. 

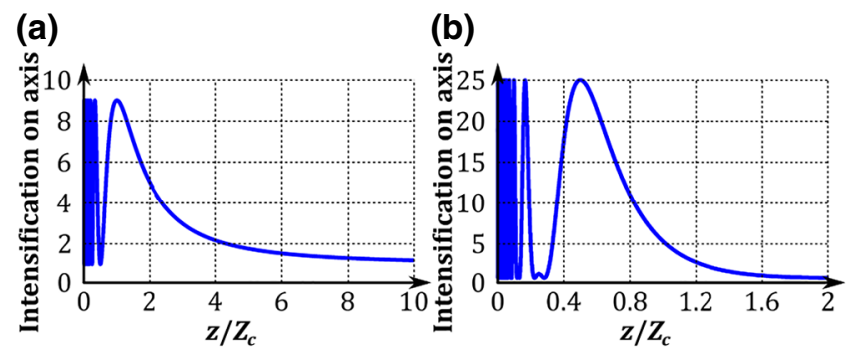

FIG. 2. Intensification along the optical axis; light propagation through a $\pi$-phase disk (a); through a phase ring $\left(\phi_{1}=0, \phi_{2}=\pi\right)(\mathrm{b})$.

\section{IMPACT OF PARTIAL PARAMETER KNOWLEDGE}

The model developed in Sec. II requires knowledge of certain parameters, such as the number of elementary disks, the transverse dimensions, the transmittances, and the phase shifts. However, some of these parameters may be impossible to measure (for instance, if the defect occurs during operation of the optical component in the laser chain). Moreover, characterizing all these parameters in an accurate way for all defects will not provide an effective defect specification because it will imply spending a huge amount of time on several instruments. Therefore, it is important to understand information provided by partial sets of parameters. We want to define safety areas, which are areas where the intensification will never be greater than a given value. These safety areas are calculated thanks to the intensification envelopes, which are computed for each partial set of parameters.

In this section, the sets of parameters are sorted with regard to characterization difficulty. Therefore, we first consider the outer radius of the defect only, which can be characterized by photography [30] (Sec. III A). In a second step, we consider, in addition, the inner radii, which can be measured with higher-resolution photography [30] (Sec. III B). Section III C deals with the simplification of the defect structure. Indeed, the defect model is structured by circular and concentric elementary objects. Moreover, discontinuous phase steps are considered. Real diffracted intensifications may have a different behavior when the defect is more complex. In Sec. III C, diffraction by structures with quasicircular shapes will be described. Finally, the phases are introduced in Sec. III D. These will need a more elaborate measurement method (such as interferometry) [30]. Of course, if all the phase shifts are known, then the model expressed by Eq. (9) predicts the diffracted intensification. The objective of Sec. III D is to see how one can take advantage of partial phase knowledge.

\section{A. Defect transverse dimension (outer radius)}

In this section, we consider that the outer radius of the defect is the only parameter that we can access. Then, we are looking for the object of outer radius $R$, which gives the highest downstream intensification for an incident plane wave at a given propagation distance $z$. Despite the fact that we considered in Sec. II constant phases over rings, we will here search for the worst phase profile without any constraints.

The maximum of intensification at distance $z$ is obtained when the phase of the diffractive contributions $U_{\text {lens }}(x, y, 0) \exp \left[j k\left(x^{2}+y^{2}\right) /(2 z)\right]$ of Eq. (7) is constant inside the defect. Moreover, we must maximize the sum of all these contributions with the field outside the defect (plane wave). This leads to the following defect wave:

$$
U_{\text {lens }}(r, \Phi)= \begin{cases}e^{-j \frac{\pi r^{2}}{\lambda z}} e^{j \Phi}, & r \in[0, R], \\ 1, & r>R,\end{cases}
$$

where $\Phi$ is a phase offset that must be tuned to maximize the interference. Therefore, if we consider the Fresnel transform in Eq. (7), we obtain

$$
\begin{aligned}
\mathfrak{D}_{(0,0, z)}\left[U_{\text {lens }}\right] & =\frac{-j k}{z} e^{j k z}\left[\int_{R}^{+\infty} e^{j\left[\pi r^{2} /(\lambda z)\right]} r d r+\int_{0}^{R} e^{j \Phi} r d r\right] \\
& =e^{j k z}\left[e^{j \pi\left(Z_{c} / z\right)}-j \pi \frac{Z_{c}}{z} e^{j \Phi}\right] .
\end{aligned}
$$

Then, the maximal intensification $I_{\text {lens }}$ at distance $z$ is given by Eq. (16) where the maximizing value of $\Phi$ is given by Eq. (17):

$$
\begin{aligned}
I_{\text {lens }} & =\max _{\Phi \in \mathbb{R}}\left|\mathfrak{D}_{(0,0, z)}\left[U_{\text {lens }}\right]\right|^{2} \\
& =\max _{\Phi \in \mathbb{R}}\left[1+\left(\pi N_{F}\right)^{2}-2 \pi N_{F} \sin \left(\pi N_{F}-\Phi\right)\right]
\end{aligned}
$$

and

$$
\Phi \equiv \pi\left(N_{F}+\frac{1}{2}\right), \quad \bmod (2 \pi)
$$

Therefore, with only the outer radius of the defect, an upper bound of the intensification $I_{R}$ can be given

$$
\widetilde{I}_{R}=\left(1+\pi N_{F}\right)^{2} .
$$

A singularity is observed at $z=0$. However, the Fresnel diffraction theory used to plot Fig. 3 applies only for $N_{F} \ll$ $R / \lambda$ or $z / Z_{c} \gg \lambda / R$, explaining why this singularity is not physical.

\section{B. Impact of the inner radii}

Taking into account the inner radii of the defect (corresponding to the internal structure) corresponds to the sequence $\left(\alpha_{i}\right)_{i \in[1, m]}$ of the model shown in Sec. II. The 


\section{(a)}

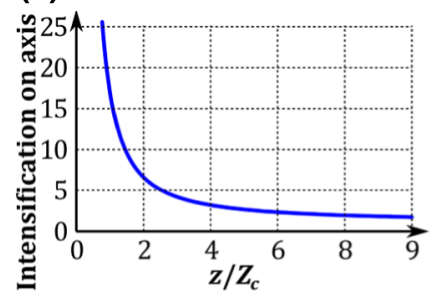

(b)

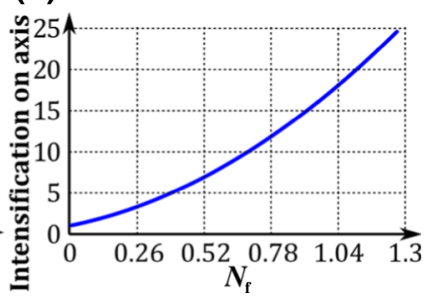

FIG. 3. Intensification envelope (namely, the $R$ envelope) given when only the defect transverse dimension is known. The $R$ envelope is plotted vs the dimensionless propagation distance $z / Z_{c}$ in (a) and vs the Fresnel number $N_{F}$ in (b).

goal is to find how the intensification envelopes depend on $\left(\alpha_{i}\right)_{i \in[1, m]}$.

First, we consider the case of a phase ring ( $m=$ $\left.2 ; t_{1}=t_{2}=t_{3}=1 ; \alpha_{1}=\alpha ; \alpha_{2}=1\right)$. The intensification $I_{\text {ring }}$ diffracted by a phase ring is computed using Eq. (9):

$$
\begin{aligned}
I_{\text {ring }}\left(N_{F}\right)= & \mid 1+2 \sin \left(\frac{\pi N_{F}}{2}(1-\alpha)\right) e^{j\left(\frac{\pi N_{F}}{2}(\alpha-1)+\phi_{2}-\frac{\pi}{2}\right)} \\
& +\left.2 \sin \left(\frac{\pi N_{F}}{2} \alpha\right) e^{j\left(\frac{\pi N_{F}}{2}(\alpha-2)+\phi_{1}-\frac{\pi}{2}\right)}\right|^{2} \text {. (19) }
\end{aligned}
$$

The two phase shifts $\phi_{1}$ and $\phi_{2}$ are isolated in the argument of complex numbers of Eq. (19). Therefore, by applying the triangular inequality, the $\left(R_{2}, \alpha\right)$ envelope, which does not depend on phase shifts, is expressed as follows:

$$
\widetilde{I}_{\alpha}\left(N_{F}\right)=\left[1+2\left|\sin \frac{\pi N_{F}}{2}(1-\alpha)\right|+2\left|\sin \frac{\pi N_{F}}{2} \alpha\right|\right]^{2} .
$$

Actually, for each Fresnel number $N_{F}$, there is a ring with particular phase shifts $\left(\phi_{1}, \phi_{2}\right)$, where the intensification $I_{\text {ring }}$ reaches the value of $\widetilde{I}_{\alpha}$ at $N_{F}$ :

$$
\begin{aligned}
& I_{\text {ring }}=\widetilde{I}_{\alpha} \\
& \Leftrightarrow\left\{\begin{array}{l}
\phi_{1}=\frac{\pi N_{F}}{2}(2-\alpha)+\frac{\pi}{2} \operatorname{sign}\left[\sin \frac{\pi N_{F}}{2} \alpha\right] \\
\phi_{2}=\frac{\pi N_{F}}{2}(1-\alpha)+\frac{\pi}{2} \operatorname{sign}\left[\sin \frac{\pi N_{F}}{2}(1-\alpha)\right]
\end{array}\right.
\end{aligned}
$$

For small $N_{F}$ values, the sinus can be linearized:

$$
\widetilde{I}_{\alpha}=\widetilde{I}_{R_{2}}+o\left(N_{F}^{2}\right)
$$

The $\left(R_{2}, \alpha\right)$ envelope developed for the phase rings case is equivalent to the $R_{2}$ envelope given by Eq. (18) for long propagation distances.

Finally, it is possible to apply Eq. (20) to the case of phase disks $\left(m=1 ; t_{1}=t_{2}=1\right)$. Indeed, a phase disk

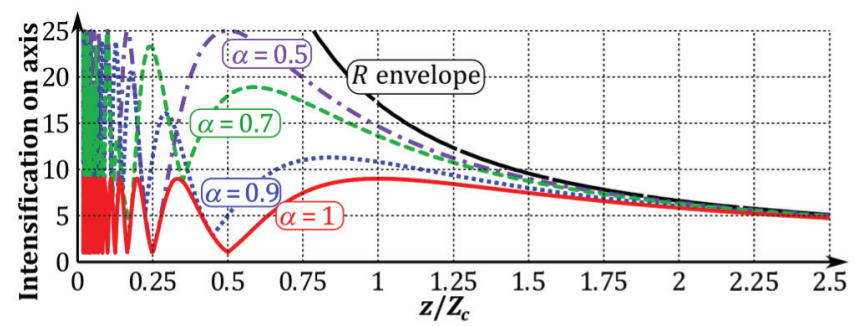

FIG. 4. Envelopes for phase rings and phase disks plotted vs dimensionless propagation distances $z / Z_{c}$ for four different $\alpha$ values.

is a phase ring where the inner radius is equal to the outer radius $(\alpha=1$ or $\alpha=0)$. Therefore, the envelope for a phase disk has the following expression:

$$
\widetilde{I}_{\alpha=1}\left(N_{F}\right)=\left[1+2\left|\sin \frac{\pi N_{F}}{2}\right|\right]^{2} .
$$

The $\left(R_{2}, \alpha\right)$ envelopes are represented in Fig. 4 for different values of $\alpha$ ( $\alpha=1$ is the envelope of the intensifications diffracted by a phase disk). Only the $\left(R_{2}, \alpha\right)$ envelopes corresponding to $\alpha$ values greater than 0.5 are represented because the $1-\alpha$ case gives the same envelope.

When the structure has elements with large differences in transverse dimensions, the contributions of the small elements do not interfere with the large ones for small $N_{F}$ values. Indeed, when $\pi N_{F} \alpha \ll 2$, Eq. (20) becomes

$$
\begin{aligned}
\tilde{I}_{\alpha}= & {\left[1+2\left|\sin \frac{\pi N_{F}}{2}\right|+\pi N_{F} \alpha\left(1-\cos \frac{\pi N_{F}}{2}\right)\right.} \\
& \left.+o\left(\frac{\left(\pi N_{F} \alpha\right)^{2}}{4}\right)\right]^{2} .
\end{aligned}
$$

In the case where $\pi N_{F} \alpha \rightarrow 0$, the $\left(R_{2}, \alpha\right)$ envelope $\widetilde{I}_{\alpha}$ has the same expression as the envelope of the intensifications diffracted by the phase disks [Eq. (23)]. The small structure in the ring has no impact on the intensifications for long propagation distances. For instance, the $\left(R_{2}, \alpha=0.04\right)$ envelope is represented by the solid line of Fig. $5\left(R_{2}\right.$ is 5 times larger than $R_{1}$ ). The dashed line, which represents the disk's envelope, has the same behavior as the ring's envelope when $z / Z_{c}$ is large enough $\left(z / Z_{c} \gg 0.1\right)$.

As the $\alpha$ and $1-\alpha$ cases are the same [Eq. (20)], the previous result established for $\alpha$ close to 0 must be extended to cases where $\alpha$ is close to 1 . Actually, in the particular case where $\alpha$ is close to 1 , the ring is so narrow that it does not contribute to the diffraction pattern for small $N_{F}$.

\section{Impact of quasicircularity}

Let us show that the model still works with quasicircular elements. The shape of the $i$ th element is now defined by 


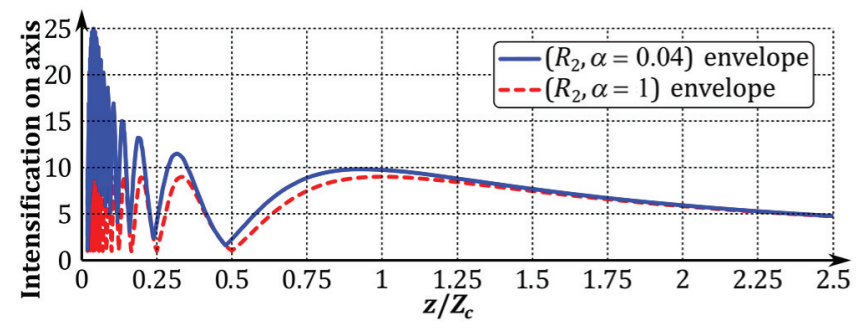

FIG. 5. Comparison between the phase rings' intensification envelope (solid line) setting $\alpha=0.04$ and the phase disk's envelope (dashed line).

$\rho_{i}$ the distance between its edge and the barycenter of the structure. It depends on the azimuthal angle $\theta$ :

$$
\rho_{i}^{2}(\theta)=R_{i}^{2}+\delta_{i}(\theta),
$$

where $R_{i}$ is the root-mean-square distance. $\delta_{i}$ is assumed to be a random variable, which follows the normal distribution $\Delta_{i} \sim \mathcal{N}\left(0, \sigma_{i}^{2}\right)$. Thus, the $i$ th element is represented by this light wave:

$$
U_{i}(r, \theta, 0)= \begin{cases}0 & \text { if } r<\rho_{i}(\theta) \\ 1 & \text { else }\end{cases}
$$

Therefore, in the Fresnel approximation, $\mathfrak{D}_{(0,0, z)}\left(U_{i}\right)$ in Eq. (5) is calculated with the stationary phase approximation:

$$
\begin{aligned}
\mathfrak{D}_{(0,0, z)}\left[U_{i}\right] & =e^{j k z} e^{j \pi N_{F}} \frac{1}{2 \pi} \int_{0}^{2 \pi} e^{j \frac{\pi \delta_{i}(\theta)}{\lambda z}} d \theta \\
& =e^{j k z} e^{j \pi N_{F}} \int_{-\infty}^{\infty} p_{\Delta_{i}}\left(\delta_{i}\right) e^{j \frac{\pi}{\lambda z} \delta_{i}} d \delta_{i},
\end{aligned}
$$

where $p_{\Delta_{i}}$ is the (Gaussian) probability density function of $\Delta_{i}$. Following [31], one can recognize in Eq. (27) the characteristic function $M_{\Delta}$ of $\Delta$, which, in this case, yields

$$
M_{\Delta}(\omega)=\int_{-\infty}^{\infty} p_{\Delta_{i}}\left(\delta_{i}\right) e^{j \omega \delta_{i}} d \delta_{i}=\exp \left(-\sigma_{i}^{2} \omega^{2} / 2\right) .
$$

Therefore, Eq. (27) becomes

$$
\begin{aligned}
\mathfrak{D}_{(0,0, z)}\left[U_{i}\right] & =e^{j k z} e^{j \pi N_{F}} e^{-(1 / 2)\left[\pi \sigma_{i} /(\lambda z)\right]^{2}} \\
& \Rightarrow I_{i}=\exp \left[-\left(\pi \frac{\sigma_{i}}{R_{i}^{2}} \frac{R_{i}^{2}}{\lambda z}\right)^{2}\right] .
\end{aligned}
$$

In the case of a quasicircular structure, Eq. (9) becomes

$$
\begin{aligned}
& \mathfrak{D}_{(0,0, z)}\left[U_{\text {str }}\right]=e^{j k z}\left[t_{1} e^{j \phi_{1}}\right. \\
& \left.+\sum_{i=1}^{m}\left(t_{i+1} e^{j \phi_{i+1}}-t_{i} e^{j \phi_{i}}\right) e^{j \pi \alpha_{i} N_{F}} K_{i}^{\alpha_{i}{ }^{2}}\right],
\end{aligned}
$$

where $K_{i}=\exp \left[-\left(\pi N_{F} \sigma_{i} / R_{i}^{2}\right)^{2} / 2\right]$.
Equation (29) shows that the intensification yielded by a quasicircular screen decreases to 0 for short propagation distances. This is not the case for the light-blocking disk. This result can be extended to the defect model. Now, the model is decomposed into phase-shifted plane waves blocked by quasicircular screens. As the Arago spot generated by the screens disappears for short propagation distances, the interferences between the elementary waves must also disappear. Therefore, intensifications along the optical axis of the model decrease for short propagation distances. For instance, the $(R, \alpha=1, \sigma)$ envelope of quasicircular phase disks may be expressed as

$$
\begin{aligned}
I_{\text {disk }} & =\left|e^{j \phi}\left(1-e^{j \pi N_{F}} K\right)+K\right|^{2} \\
& <\left(\left|1-e^{j \pi N_{F}} K\right|+K\right)^{2} .
\end{aligned}
$$

The $(R, \alpha=1, \sigma)$ envelope tends to 1 for high $N_{F}$ values instead of the perfectly circular case limited by the $\left(R_{2}, \alpha=1\right)$ envelope [see Eq. (23)], which predicts continuous oscillations of the intensifications for high $N_{F}$ values.

In the particular case of a phase ring, the impact of a quasicircular shape is given by the two terms: $\left|\mathfrak{D}_{(0,0, z)}\left[U_{2}\right]\right|$ and $\left|\mathfrak{D}_{(0,0, z)}\left[U_{1}\right]\right|$. Actually, when the quantities $\sigma_{i} / R_{i}^{2}$ are the same for the two elements $\left(K_{1}=K_{2}=K\right)$, we get

$$
\left\{\begin{array}{l}
\left|\mathfrak{D}_{(0,0, z)}\left[U_{2}\right]\right|=K \\
\left|\mathfrak{D}_{(0,0, z)}\left[U_{1}\right]\right|=K^{\alpha^{2}}
\end{array} \quad \Rightarrow \quad \frac{\left|\mathfrak{D}_{(0,0, z)}\left[U_{2}\right]\right|}{\left|\mathfrak{D}_{(0,0, z)}\left[U_{1}\right]\right|}=K^{1-\alpha^{2}}\right.
$$

When the phase ring has elements with a large difference in transverse dimensions $(\alpha \ll 1)$, then $K^{1-\alpha^{2}} \approx K$. Moreover, for short propagation distances $\left(\pi N_{F} \gg R_{i}^{2} / \sigma_{i}\right)$, the term $K$ is close to zero, which means that the large element no longer contributes to the diffraction pattern. With these assumptions, Eq. (30) applied to the ring becomes

$$
\begin{aligned}
I_{\text {ring }}\left(N_{F} \gg \frac{R_{2}^{2}}{\pi \sigma_{2}}\right) \approx & \mid e^{j \phi_{1}}\left(1-K^{\alpha^{2}} e^{j \pi \alpha N_{F}}\right) \\
& +\left.K^{\alpha^{2}} e^{j \pi \alpha N_{F}} e^{j \phi_{2}}\right|^{2} .
\end{aligned}
$$

According to the triangular inequality, Eq (33) becomes

$$
I_{\text {ring }}<\left(\left|1-K^{\alpha^{2}} e^{j \pi \alpha N_{F}}\right|+K^{\alpha^{2}}\right)^{2} .
$$

For short propagation distances, the ring's envelope with small $\alpha$ is the same as the envelope of the small element [Eq. (31)]. Therefore, for small $\alpha$ values and when the propagation distance is short enough, the large element no longer interferes with the small one.

For instance, in the case of Sec. III B (envelope of phase rings with $\alpha=0.04$ ), some circular imperfections are 


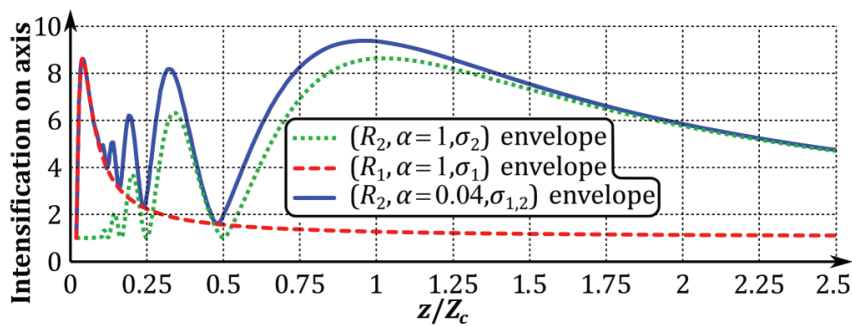

FIG. 6. Intensification envelopes downstream of specific noncircular phase objects $\left(\alpha=0.04 ; \sigma_{1} / R_{1}^{2}=0.08 ; \sigma_{2} / R_{2}^{2}=0.08\right)$. The solid line is the envelope of the quasicircular rings $\left(R_{1}^{2}=\alpha R_{2}^{2}\right)$, the dashed line is the envelope of the quasicircular disks $\left(R=R_{1}\right)$, and the green dotted line is the envelope of the quasicircular disks $\left(R=R_{2}\right)$.

added: $\sigma_{1} / R_{1}^{2}=\sigma_{2} / R_{2}^{2}=0.08$. The three envelopes with the circularity considerations are represented in Fig. 6: the solid line is the $\left(R_{2}, \alpha=0.04, \sigma_{1,2}\right)$ envelope computed for the ring, the dotted line is the $\left(R_{2}, \alpha=1, \sigma_{2}\right)$ envelope of the outer radius disks, and the dashed line is the $\left(R_{1}, \alpha=1, \sigma_{1}\right)$ envelope of the inner radius disks. The solid and the dashed lines are merged: the large element does not interfere with the small one for short propagation distances $\left[N_{F} \gg R^{2} /(\pi \sigma)\right]$.

\section{Impact of partial phase knowledge}

The intensification envelopes we have defined never took into consideration the phases of the diffracting structure. The general idea of these envelopes is that the larger the defect transverse dimension, the farther the safety area is downstream of the defect. In this section, we show how partial knowledge of phase shifts provides envelopes that improve information about the safety area.

In the phase-ring case, two envelopes are developed from the knowledge of only one of the two phase shifts. Indeed, instead of isolating in Eq. (19) the two phase shifts in the arguments of the complex exponentials to obtain the $(R, \alpha)$ envelope described by Eq. (20), only the phase $\phi_{2}$ can be isolated to get an envelope depending on the phase $\phi_{1}$,

$$
\begin{aligned}
\widetilde{I}_{\alpha, \phi_{1}}= & {\left[1+2\left|\sin \frac{\pi N_{F}}{2}(1-\alpha)\right|\right.} \\
& \left.+2\left|\sin \left(\frac{\pi N_{F}}{2}(1-\alpha)-\frac{\phi_{1}}{2}\right)\right|\right]^{2} .
\end{aligned}
$$

The ( $\left.R, \alpha, \phi_{1}\right)$ envelope expressed by Eq. (35) is interesting to use in cases when $\alpha$ is close to 1 . In the same way, we can obtain an envelope depending on the phase $\phi_{2}$, which is useful when $\alpha$ is close to zero.

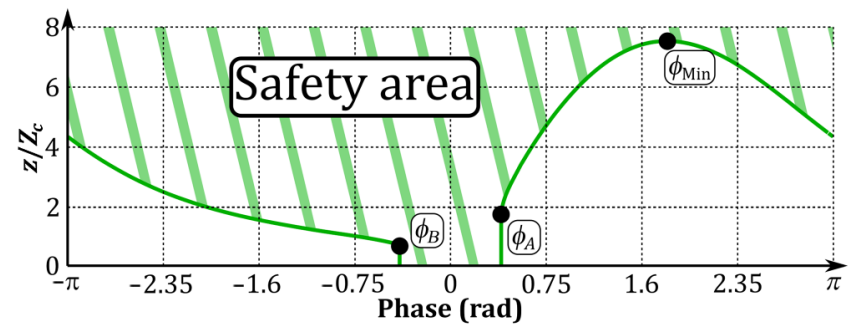

FIG. 7. Safety distances downstream of phase disks vs phase shifts of the disk. The intensifications remain lower than $I_{0}$ in the safety area $\left(I_{0}=2\right)$.

Finally, for the particular case of a phase disk, the diffracted intensification is less complex,

$$
I_{\text {disk }}=1+4 \sin ^{2} \frac{\phi}{2}+4 \sin \frac{\phi}{2} \sin \left(\pi N_{F}-\frac{\phi}{2}\right) .
$$

The safety area of a phase disk can be evaluated as follows. Let us represent the safety area by a distance $z_{0} / Z_{c}$ corresponding to the shortest distance where the diffracted intensification will never be higher than a given value $I_{0}\left(I_{\text {disk }}\left(z>z_{0}\right)<I_{0}\right)$. These "safety distances" are shown in Fig. 7 with regard to the phase shift $\left(I_{0}=2\right)$. Three particular phase shifts appear:

$$
\left\{\begin{array}{l}
\phi_{A}=-\phi_{B}=2 \arcsin \frac{\sqrt{I_{0}}-1}{2} \\
\phi_{\text {Min }}=\frac{\phi_{A}+\pi}{2}
\end{array}\right.
$$

Between $\phi_{B}$ and $\phi_{A}$, the phase is too small and the intensification cannot be higher than $I_{0}$. Therefore, whatever the transverse dimension, if the phase shift belongs to $\left[\phi_{B}, \phi_{A}\right]$, the defect is not dangerous. The point $\phi_{\mathrm{Min}}$ is the phase associated with the farthest safety distance. In fact, it corresponds to the safety distance that can be established in the $(R, \alpha=0)$ envelope case [Eq. (23)] where only the transverse dimension is known.

Finally, it is remarkable that positive phases generate safety areas farther than negative phases. This proves that for optical transmission components, under the assumption of phases ranging between $[-\pi, \pi]$, bump-shape defects are more dangerous than hole-shape defects.

\section{COMPARISON WITH EXPERIMENTAL RESULTS}

The intensifications diffracted by three particular defects are now investigated experimentally. Defect 1 ensues from pollution by a drop of solvent, defect 2 is an AR bump caused by a particle on the substrate during AR coating deposition, while defect 3 is the mitigation of a laserinduced damage site made by using a $\mathrm{CO}_{2}$ laser [7] 
TABLE I. Parameters of defect 1 characterized by interferometry.

\begin{tabular}{lccc}
\hline \hline \multicolumn{2}{l}{ Parameters } & Interior ring $(i=1)$ & Exterior ring $(i=2)$ \\
\hline$R_{i}(\mu \mathrm{m})$ & Nomarski & 98 & 105 \\
& Profiler & 93 & 101 \\
$\sigma_{i} / R_{i}^{2}$ & Nomarski & 0.023 & 0.035 \\
& Profiler & 0.019 & 0.030 \\
$\phi_{i}(\mathrm{rad})$ Profiler & 0.158 & 1.063 \\
\hline \hline
\end{tabular}

(Defects 1 and 2 are coating defects, AR layer with refraction index: $n_{\mathrm{AR}}=1.22$; the phase shifts will be defined by $\phi_{i}=k_{0} h_{i}\left(n_{\mathrm{AR}}-1\right)$ where $h_{i}$ are mechanical heights.). The intensifications by these three defects are measured using a setup which probes the diffracted intensity [11]. A collimated laser beam at $351 \mathrm{~nm}$ propagates through the defects and the diffracted light is measured using a CCD sensor at different propagation distances (under $1 \mathrm{~m}$ ). The intensifications are compared to the envelopes established in the previous section. These envelopes are obtained using parameters that are characterized with three types of measurements: light diffusion, Nomarski microscopy, and interferometric microscope. The first two measurements only give information on transverse dimensions $\left(R_{i}, \sigma_{i}\right)$. However, Nomarski microscopy is more sensitive to structures with low-phase shifts. In addition, the interferometric microscope measures the defect heights (phase shifts of the structure). The parameters of the three defects are given in Tables I-III.

\section{A. Defect 1: a drop on a sol-gel antireflection coating}

According to the observations shown in Fig. 8, the defect structure is a phase ring $\left(m=2 ; t_{1}=t_{2}=t_{3}=1\right.$; $\alpha_{1}=\alpha ; \alpha_{2}=1$ ). The complete model (considerations about the transverse dimensions, circularity, and phase shifts [Table I)] is shown in Fig. 9 (solid line). The model merges with the intensification measurements (circle markers).

TABLE II. Parameters of defect 2 characterized by three different setups.

\begin{tabular}{lccccc}
\hline \hline & & Particle & AR bump & \multicolumn{2}{c}{ Surrounding ring } \\
\cline { 5 - 6 } Parameters & $i=1$ & $i=2$ & $i=3$ & $i=4$ \\
\hline$R_{i}(\mu \mathrm{m})$ & Diffusion & - & 217 & - & - \\
& Nomarski & 48 & 234 & - & - \\
& Profiler & 57 & 234 & 393 & 505 \\
$\sigma_{i} / R_{i}^{2}$ & Diffusion & - & 0.12 & - & - \\
& Nomarski & 0.569 & 0.078 & - & - \\
& Profiler & 0.481 & 0.068 & 0.393 & 0.059 \\
$\phi_{i}(\mathrm{rad})$ Profiler & - & 0.52 & -0.012 & 0.040 \\
\hline \hline
\end{tabular}

TABLE III. Parameters of defect 3 characterized by optical profiler measurement.

\begin{tabular}{lcc}
\hline \hline & \multicolumn{2}{c}{ Raised rim } \\
\cline { 2 - 4 } Parameters & Interior $(i=1)$ & Exterior $(i=2)$ \\
\hline$R_{i}(\mu \mathrm{m})$ & 1.05 & 1.1 \\
$\sigma_{i} / R_{i}^{2}$ & 0.0152 & 0.0327 \\
$\phi_{i}(\mathrm{rad})$ Profiler & & 0.42 \\
\hline \hline
\end{tabular}

Moreover, thanks to the envelopes developed in Sec. III, safety distances (distance after which the diffracted intensifications do not exceed $I_{0}=2$ ) are provided and compared to the measured distance: $z_{0 \text {,mea }}=18 \mathrm{~mm}$. When only transverse dimensions and circularity are known, the safety distance is found at $237 \mathrm{~mm}$ (dashed line). It is about 10 times farther than what is measured. To improve this estimation, the only solution is to obtain some information about the phase shifts. Actually, the ratio of the square radii is close to $1(\alpha=0.88)$. Therefore, knowledge of only the inner phase shift is enough to provide a much better safety area [Eq. (35)]. The $\left(R_{2}, \alpha, \phi_{1}\right)$ envelope is represented by the dotted line and the safety distance is estimated at $z_{0 \text {,est }}=42 \mathrm{~mm}$. This shows how partial information might be enough to obtain a safety area close to reality.

\section{B. Defect 2: a bump on an antireflection coating}

Defect 2 has a complex structure. Indeed, in addition to the AR bump and the particle, a surrounding ring appears on Nomarski measurements [Fig. 10(b)]. Therefore, the number of required disks is assumed to be $m=4$.

However, using Eq. (13) and the values of Table II, the intensifications created by the surrounding ring alone cannot exceed $I_{\text {ring }}<1.2$. The phase shifts of this ring are too small to have an impact on the diffracted pattern. Thus, the surrounding ring is not considered to compute the safety area and we use $m=2$ (particle + AR bump). Moreover, regarding the transverse dimensions and the
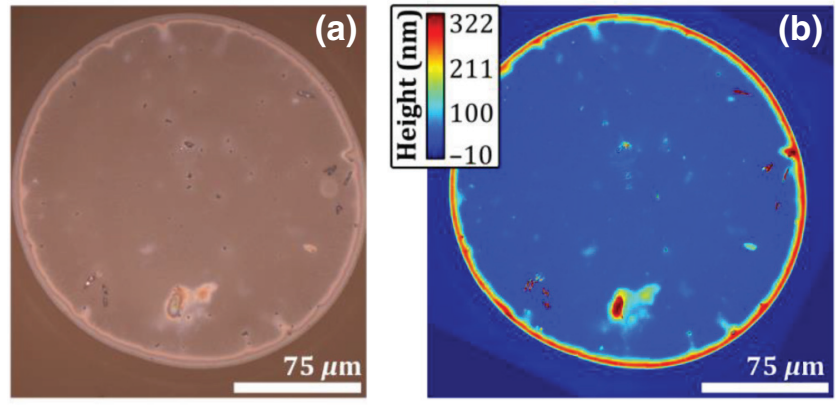

FIG. 8. Observations of defect 1; (a) Nomarski measurement; (b) phase measurement with an optical profiler. 


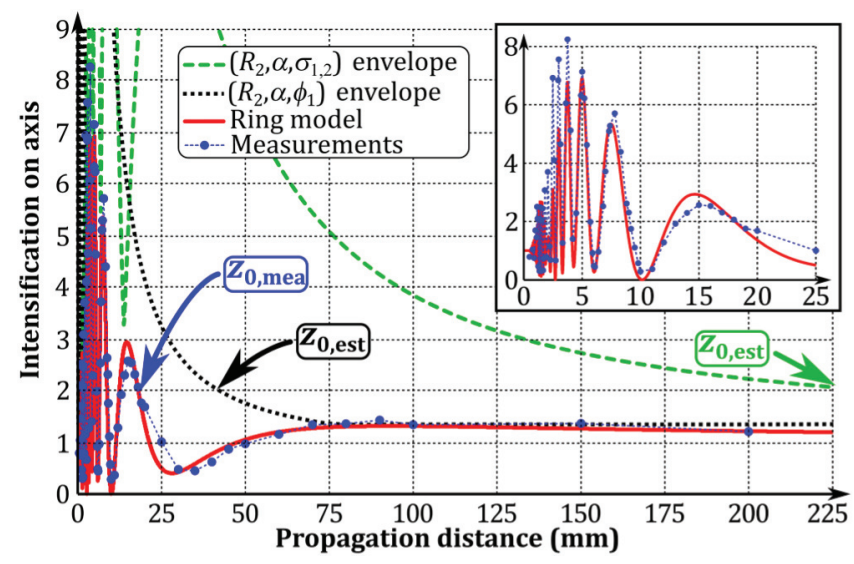

FIG. 9. Intensifications and envelopes of defect 1 vs propagation distance. When all parameters are known, the intensification (solid line) has the same behavior as the measurement (circle markers). However, even a partial phase knowledge (dotted line) gives a safety distance close to the measured distance.

circularity of the particle and the AR bump, these two elements cannot interfere as has been shown in Sec. III C $\left(\alpha=0.04 ; \sigma_{2} / R_{2}^{2}=0.08\right)$.

Despite its complex structure, defect 2 is reduced to a simple phase disk thanks to the considerations made in Sec. III. Only the AR-bump phase shift is needed. The validity of this simplification is shown in Fig. 11. Indeed, the disk model (solid red line) is very close to the measurements (circle markers).

Finally, safety distances are compared to the measured distances: $z_{0 \text {, mea }}=375 \mathrm{~mm}\left(I_{0}=2\right)$. The first envelope is computed with only the transverse dimension (dashed line). Once again, the estimation of the safety distance
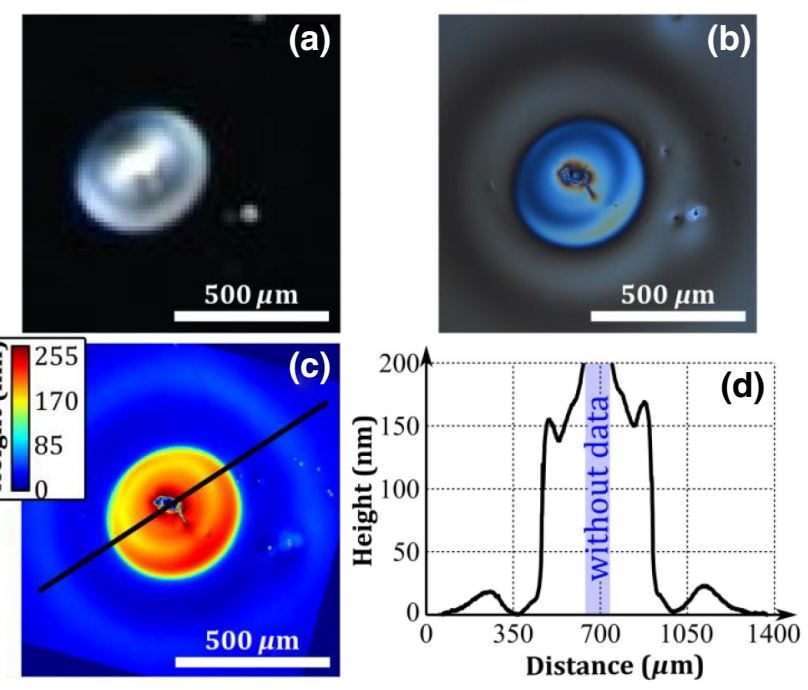

FIG. 10. Observations of defect 2; (a) light diffusion photography; (b) Nomarski measurement; (c) phase measurement with optical profiler; (d) profile along the black line.

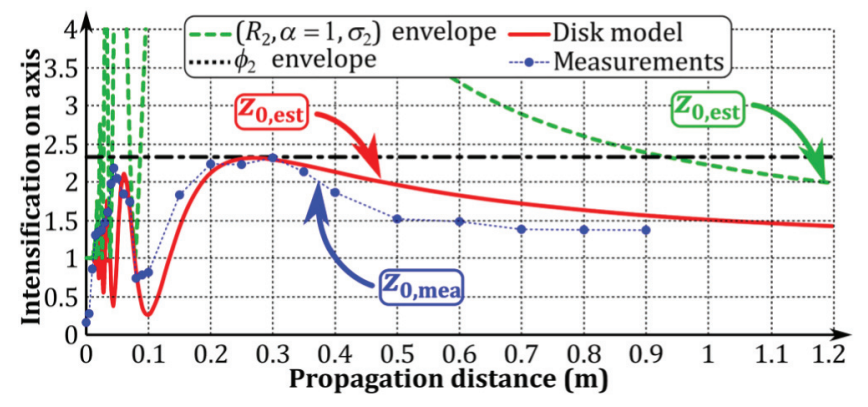

FIG. 11. Intensifications and envelopes of defect 2 vs propagation distance. The complex defect structure has the same impact on light as the phase disk.

is oversized: $1182 \mathrm{~mm}$. Information about the phase improves the estimation. Regarding the profiler measurement [Fig. 10(c)], the phase shift of the AR bump is estimated at $0.52 \mathrm{rad}$. First of all, the intensification can be limited to $I_{\text {disk }}<2.3$ thanks to Eq. (13) (dotted line). By using the phase disk model (red line), the safety distance is: $z_{0, \text { est }}=470 \mathrm{~mm}$.

\section{Defect 3: mitigation of laser-induced damage}

Defect 3 comes from the recycling process of damaged optics. This process generates an object with a conical shape that refracts light [Fig. 12]. Therefore, from a distance, it looks like no more light comes from the conical shape. It seems that the defect could be modeled by a simple light-blocking disk. However, for particular mitigations, the central intensity is higher than it should be in an Arago spot (intensity along the optical axis higher than the incident intensity) [32]. Actually, these intensifications are explained by the presence of a surrounding raised rim due to the recycling process [33]. Therefore, the number of decomposed disks is $m=2$.

The intensifications diffracted by the model are represented by the green dashed line in Fig. 13. For distances shorter than $200 \mathrm{~mm}$, there is a discrepancy between the model, which predicts oscillations, whereas the measurements drop to 0 . To understand this, the quasicircular shape (a)

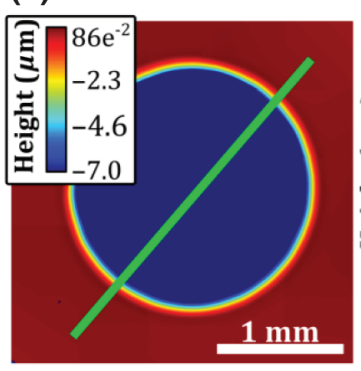

(b)

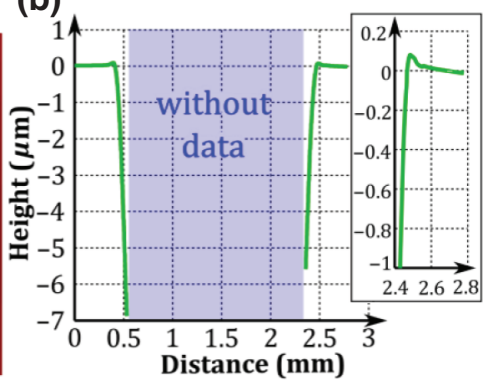

FIG. 12. Observation of defect 3; (a) phase measurement with optical profiler; (b) profile along the green line. 


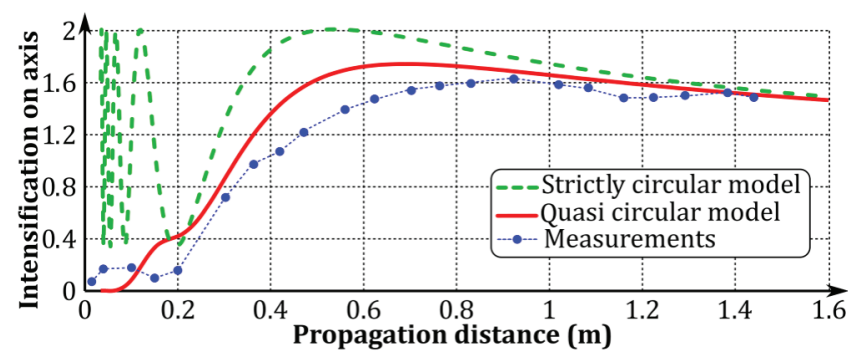

FIG. 13. Intensifications measured on defect 3 and models vs propagation distance. The quasicircular model explains the drop to 0 of intensification measurements.

of the object has to be considered. Indeed, the central interference phenomenon vanishes for short propagation distances (solid line) when the shape is no longer circular (as explained in Sec. III C).

\section{CONCLUSION}

In this paper, we theoretically and experimentally demonstrate that a defect on the surface of an optical component can be decomposed into elementary waves (a plane wave and several plane waves blocked by concentric opaque disks) with regard to its on-axis diffraction impact. Interference phenomena between these disks lead to "hot spots" on the optical axis. To know why a defect generates high intensifications, it is necessary to perform some characterizations such as the number of elementary disks, their radii, their transmittances, or their phase shifts.

However, these parameters may sometimes prove challenging to measure. They may be partially or totally unknown. Thanks to the analytic approach, we determine envelopes to intensifications in order to find safety areas as close as possible within the knowledge of a given set of parameters. Phase knowledge can be very useful to avoid overestimation of the defect impact on light. The difficulty here results from the observation of the phase shift. In this paper, we use an optical profiler to deal with this issue. However, in practical cases, the use of such an instrument can be time consuming to obtain an excessively accurate characterization. Actually, partial knowledge of the phase is enough to evaluate the defect danger on downstream optics. Therefore, phase observation can be less accurate, as with differential interference contrast methods.

Moreover, thanks to considerations about the influence of each parameter on the diffracted light, it is shown that simplifications of the defect structure can sometimes be made. Generally, it will be sufficient to decompose a defect into a maximum of two elementary disks and a phase-shifted plane wave.

We must acknowledge that the general model makes strong assumptions. The defect shape is assumed to be circular with concentric elementary disks. The phase shifts between disks have infinite slopes and the phase within an element is constant. Stepping outside this model will change the intensification behavior. We show in Sec. III D how quasicircularity modifies this behavior. The first intensifications drop to zero. These assumptions can have a huge impact, but only for short propagation distances (below the safety distance). Then, the envelopes based on the first model are enough to provide safety areas downstream of defects.

[1] A. Fresnel, Mém. Acad. Sci. (Acad. Sci., Paris, 1826), Tome 5, p. 339.

[2] Y. Zheng, Z. Liu, J. Luo, F. Pan, J. Wang, and Q. Xu, Modeling and characterization of wavefront morphologies of laser induced damages on dielectric coating, Opt. Commun. 403, 317 (2017).

[3] A. Babinet, C. R. Acad. Sci. (Acad. Sci., Paris, 1837), Tome 4, p. 644.

[4] M. Born and E. Wolf, Principles of Optics: Electromagnetic Theory of Propagation, Interference and Diffraction of Light (Pergamon Press, Oxford, 1959 [6 $6^{\text {th }}$ ed. 1980]), p. 381.

[5] M. C. Nostrand, C. J. Cerjan, M. A. Johnson, T. I. Suratwala, T. L. Weiland, W. D. Sell, J. L. Vickers, R. L. Luthi, J. R. Stanley, T. G. Parham, and C. B. Thorsness, Correlation of laser-induced damage to phase objects in bulk fused silica, Proc. SPIE 5647, 233 (2004).

[6] J. A. Menapace, P. J. Davis, W. A. Steele, M. R. Hachkowski, A. Nelson, and K. Xin, MRF applications: on the road to making large-aperture ultraviolet laser resistant continuous phase plates for high-power lasers, Proc. SPIE 6403, 64030N (2007).

[7] T. Doualle, L. Gallais, S. Monneret, S. Bouillet, A. Bourgeade, C. Ameil, L. Lamaignère, and P. Cormont, $\mathrm{CO}_{2}$ laser microprocessing for laser damage growth mitigation of fused silica optics, Opt. Eng. 56, 011022 (2016).

[8] M. J. Matthews, N. Shen, J. Honig, J. D. Brude, and A. M. Rubenchik, Phase modulation and morphological evolution associated with surface-bound particle ablation, J. Opt. Soc. Am. B 30, 3233 (2013).

[9] X. Liu, Y. Zhao, D. Li, G. Hu, Y. Gao, Z. Fan, and J. Shao, Characteristics of plasma scalds in multilayer dielectric films, Appl. Opt. 50, 4226 (2011).

[10] M. Runkel, R. Hawley-Fedder, C. Widmayer, W. Williams, C. Weinzapfel, and D. Roberts, A system for measuring defect induced beam modulation on inertial confinement fusion-class laser optics, Proc. SPIE 5991, 59912H (2006).

[11] F. Tournemenne, S. Bouillet, C. Rouyer, B. Da Costa Fernandes, and G. Gaborit, Visual defects diffraction in high power lasers: Impact on downstream optics, Proc. SPIE 10447, 104471Y (2017).

[12] M. J. Matthews, I. L. Bass, G. M. Guss, C. C. Widmayer, and F. L. Ravizza, Downstream intensification effects associated with $\mathrm{CO}_{2}$ laser mitigation of fused silica, Proc. SPIE 6720, 67200A (2007).

[13] L. Lamaignère, S. Bouillet, R. Courchinoux, T. Donval, M. Josse, J.-C. Poncetta, and H. Bercegol, An accurate, repeatable, and well characterized measurement of laser damage 
density of optical materials, Rev. Sci. Instrum. 78, 103105 (2007).

[14] S. Mainguy, B. Le Garrec, and M. Josse, Downstream impact of flaws on the LIL/LMJ laser lines, Proc. SPIE 5991, 599105 (2005).

[15] A. Bourgeade, T. Donval, L. Gallais, L. Lamaignère, and J.L. Rullier, Modeling surface defects in fused silica optics for laser wave propagation, J. Opt. Soc. Am. B 32, 655 (2015).

[16] O. Morice, Miro: Complete modeling and software for pulse amplification and propagation in high-power laser systems, Opt. Eng. 42, 1530 (2003).

[17] R. A. Stacks, K. P. McCandless, E. Feigenbaum, J. M. G. Di Nicola, K. J. Luke, W. Riedel, R. J. Learn, and B. J. Kraines, The virtual beamline (VBL) laser simulation code, Proc. SPIE 9345, 93450M (2015).

[18] Y. Yang, S. Wang, X. Chen, L. Li, P. Cao, L. Yan, Z. Cheng, and D. Liu, Sparse microdefect evaluation system for large fine optical surfaces based on dark-field microscopic scattering imaging, Proc. SPIE 8838, 883806 (2013).

[19] F. L. Ravizza, M. C. Nostrand, L. M. Kegelpeyer, R. A. Hawley, and M. A. Johnson, Process for rapid detection of fratricidal defects on optics using linescan phasedifferential imaging, Proc. SPIE 7504, 75041B (2009).

[20] O. Bryngdahl, Diffraction patterns of small phase objects measured in the microwave region, Ark. Fys 16, 69 (1959).

[21] M. Totzeck and M. A. Krumbügel, Extension of Babinet's principle and the Andrews boundary diffraction wave to weak phase objects, J. Opt. Soc. Am. A 11, 3235 (1994).

[22] S. Mainguy, I. Tovena-Pécault, and B. Le Garrec, Propagation of LIL/LMJ beams under the interaction with contamination particles, Proc. SPIE 5991, 59910G (2005).
[23] Y. Zheng, P. Ma, H. Li, Z. Liu, and S. Chen, Studies on transmitted beam modulation effect from laser induced damage on fused silica optics, Opt. Express 21, 16605 (2013).

[24] J. W. Goodman, Introduction to Fourier Optics (McGrawHill Higher Education, New York, 1968 [2nd ed. 1996]).

[25] N. Bleistein and R. A. Handelsman, Asymptotic Expansion of Integrals (Holt, Rinehart and Winston, New York, 1975).

[26] J. J. Stamnes, Uniform asymptotic theory of diffraction by apertures, J. Opt. Soc. Am. 73, 96 (1983).

[27] W. H. Southwell, Validity of the Fresnel approximation in the near field, J. Opt. Soc. Am. 71, 7 (1981).

[28] F. D. Feiock, Wave propagation in optical systems with large apertures, J. Opt. Soc. Am. 68, 485 (1978).

[29] M. Born and E. Wolf, Principles of Optics: Electromagnetic Theory of Propagation, Interference and Diffraction of Light (Pergamon Press, Oxford, 1959 [6 ${ }^{\text {th }}$ ed. 1980]), p. 371.

[30] J. Daurios, S. Bouillet, G. Gaborit, and J. C. Poncetta, Optical metrology devices for high-power laser large optics, Proc. SPIE 6616, 661645 (2007).

[31] J. W. Goodman, Statistical Optics (John Wiley \& Sons, New York, 1985), p. 19.

[32] G. Guss, I. Bass, V. Draggoo, R. Hackel, S. Payne, M. Lancaster, and P. Mak, Mitigation of growth of laser initiated surface damage in fused silica using a 4.6-micron wavelength laser, Proc. SPIE 6403, 64030M (2006).

[33] I. L. Bass, G. M. Guss, M. J. Nostrand, and P. L. Wegner, An improved method of mitigating laser-induced surface damage growth in fused silica using a rastered pulsed $\mathrm{CO}_{2}$ laser, Proc. SPIE 7842, 784220 (2010). 